\title{
Hypoxaemia during pregnancy: pulmonary arteriovenous dilatation as a likely cause
}

\author{
To the Editor:
}

Pregnancy induces marked physiological changes in the respiratory and cardiovascular systems that can be accompanied by "physiological dyspnoea", but not by any significant modification of haematosis [1].

Herein, we report the case of a 34-year-old pregnant female at 22 weeks gestation of spontaneous dichorionic diamniotic twins, who was referred to the emergency room for increasing dyspnoea. She had a smoking history of 15 pack-years. She had had a caesarean delivery for her first pregnancy 5 years earlier, but had not suffered any dyspnoea. She had no personal or family history of either thromboembolic disease or epistaxis. Dyspnoea had started at the beginning of the current pregnancy and had recently increased (grade 2 of the modified Medical Research Council scale). The patient had no fever, cough or chest pain. At examination, she had palmar erythema and telangiectasia on the chest, which had appeared 3 months earlier. She had no signs of heart failure or thrombophlebitis of the legs. Cardiopulmonary auscultation was normal.

Leukocyte count, haemoglobin level and brain natriuretic peptide were normal. D-dimers were elevated $\left(1158 \mathrm{ng} \cdot \mathrm{mL}^{-1} ; \mathrm{n}<500\right)$. Arterial blood gas at rest in the supine position and in ambient air showed an increased alveolar-arterial oxygen tension difference $\left(P \mathrm{~A}-\mathrm{aO} \mathrm{O}_{2}\right)$, since arterial carbon dioxide tension $\left(\mathrm{PaCO}_{2}\right)$ was $26 \mathrm{mmHg}$ and arterial oxygen tension $\left(\mathrm{PaO}_{2}\right)$ was $72 \mathrm{mmHg}$. The ECG was normal but in sinus tachycardia $\left(96\right.$ beats $\cdot \mathrm{min}^{-1}$ ). Clinical probability of pulmonary embolism was considered moderate. Doppler ultrasound of the legs, computed tomography (CT) scan, ventilation/perfusion lung scan and transthoracic Doppler echocardiography were normal and ruled out pulmonary embolism. The CT scan also showed normal lung parenchyma.

On pulmonary function tests (PFTs), lung volumes and flow rates were normal. By contrast, transfer of the lung for carbon monoxide (TLCO) was dramatically decreased (34\% predicted). An intracardiac right-to-left shunt was suspected, and a hyperoxia test (arterial blood gases breathing 100\% oxygen for 30 min) was performed in the upright position. $\mathrm{PaO}_{2}$ increased to $520 \mathrm{mmHg}$ (and $\mathrm{PaCO}_{2}$ to $35 \mathrm{mmHg}$ ), rendering a true shunt unlikely. Dyspnoea and hypoxaemia worsened during the following 4 weeks. Orthodeoxia was observed, since $\mathrm{PaO}_{2}$ decreased from $62 \mathrm{mmHg}$ to $54 \mathrm{mmHg}$ when the patient moved from the supine to the upright position. Transthoracic contrast echocardiography showed a large volume of bubbles entering the left atrium four cardiac cycles after opacification of the right atrium. Abdominal Doppler echography did not reveal any sign of portal hypertension. The diagnosis of pulmonary arteriovenous dilatation was retained as the most probable. Supplemental oxygen and prophylactic antithrombotic therapy were given and close fetal monitoring was performed. At 28 weeks gestation, the situation had deteriorated further, with increased dyspnoea and hypoxaemia $\left(\mathrm{PaO}_{2} 48 \mathrm{mmHg}\right.$ in the supine position breathing room air). The hyperoxia test was repeated in the supine position $\left(\mathrm{PaO}_{2} 310 \mathrm{mmHg}\right)$. Contrast transthoracic echocardiography still excluded both intracardiac shunting (bubbles seen after five to six cardiac cycles) and pulmonary hypertension.

Caesarean delivery was performed at 32 weeks gestation. The twins were eutrophic. During the immediate postpartum period, hypoxaemia and orthodeoxia persisted. Transoesophageal contrast Doppler echocardiography confirmed massive extracardiac shunting, without any intracardiac defect (fig. 1). The absence of intracardiac defect was confirmed by right heart catheterisation, which also showed normal pulmonary haemodynamics (pulmonary artery pressure: $22 / 8-14 \mathrm{mmHg}$; cardiac index: $3.2 \mathrm{~L} \cdot \mathrm{min}^{-1} \cdot \mathrm{m}^{-2}$; pulmonary artery occlusion pressure: $8 \mathrm{mmHg}$; right atrial pressure: $3 \mathrm{mmHg}$; mixed venous oxygen saturation: $80 \%$ ). Pulmonary angiography did not show any large fistula.

4 months after delivery, dyspnoea had largely improved (modified Medical Research Council grade 1). PFTs showed persistent low TLCO (42\% predicted). A cardiopulmonary exercise test (CPET) with evaluation of cardiac output during exercise with a thoracic impedance device showed increased ventilatory equivalents (minute ventilation $\left(V^{\prime} \mathrm{E}\right)$ /oxygen uptake $\left(V^{\prime} \mathrm{O}_{2}\right): 52 ; V^{\prime} \mathrm{E} /$ carbon dioxide uptake $\left(V^{\prime} \mathrm{CO}_{2}\right): 43$ at peak exercise) and oxygen desaturation at peak exercise from $97 \%$ to $91 \%$, but was otherwise normal. A rehabilitation programme without supplemental oxygen was initiated. 4 months later the patient had no dyspnoea, improved TLCO (59\% predicted), normal blood gases at rest $\left(\mathrm{PaCO}_{2}: 38 \mathrm{mmHg} ; \mathrm{PaO}_{2}: 93 \mathrm{mmHg}\right)$, normal 

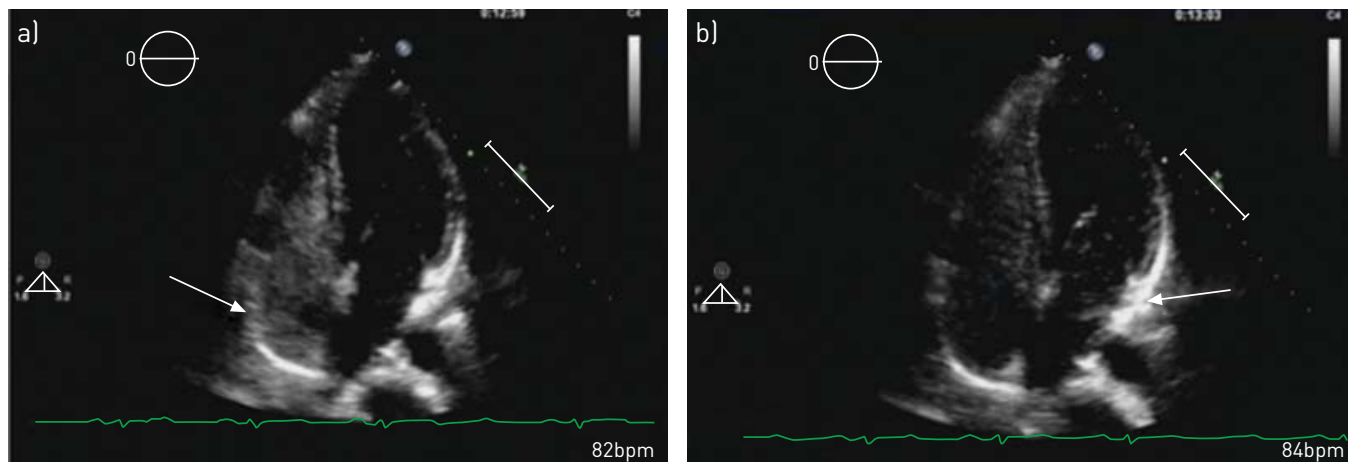

FIGURE 1 Saline contrast-enhanced transthoracic echocardiography showing a positive contrast test for intrapulmonary vascular dilatation diagnosis. Arrows indicate microbubbles inside the a) right atrium and b) just after transit through the left atrium. Microbubbles entered the left atrium $3.5 \mathrm{~s}$ (four to five cardiac cycles) after they passed through the right atrium. bpm: beats per minute.

Doppler echocardiography and normal CPET $\left(V^{\prime} \mathrm{E} / V^{\prime} \mathrm{O}_{2}: 36 ; V^{\prime} \mathrm{E} / V^{\prime} \mathrm{CO}_{2}: 33\right.$ at peak exercise) without oxygen desaturation.

The diagnosis of transient pulmonary arteriovenous dilatation was retained as being the most plausible diagnosis. The vascular effects of hormonal changes related to pregnancy were considered the most likely cause.

Numerous conditions are associated with a decrease of both $\mathrm{PaO}_{2}$ and $\mathrm{PaCO}_{2}$ [2]. Pulmonary embolism and pneumonia are frequent during pregnancy but were excluded here [3]. The very low TLCO value, along with otherwise normal PFTs, was unlikely to be caused by abnormal membrane conductance in the absence of any abnormality on the CT scan [4]. Low capillary blood volume is observed in pulmonary embolism and pulmonary arterial hypertension; these diagnoses were excluded in this case. A low TLCO is also found in hepatopulmonary syndrome [5]. The late appearance of bubble contrast in the left side of the heart on echocardiography ruled out an intracardiac shunt and suggested transit of the bubble contrast through pulmonary arteriovenous fistulae. Large pulmonary arteriovenous fistulae are a hallmark of haemorrhagic hereditary telangiectasia, a disease that is prone to deterioration during pregnancy [6]. However, haemorrhagic hereditary telangiectasia causes a true shunt, and the first hyperoxia test performed in our patient excluded a true shunt. Although hepatopulmonary syndrome was excluded by definition in the absence of portal hypertension, a perfusion-diffusion defect, as found in hepatopulmonary syndrome, was the most likely cause of hypoxaemia in our patient $[2,7]$. The association of a marked increase in $P \mathrm{~A}-\mathrm{aO}_{2}$ when breathing room air, together with the measurement of a small shunt effect when breathing $100 \%$ oxygen is the functional hallmark of this anomaly. The fact that blood is perfused in fewer over-dilated alveolar-capillary units than normal also explains the low TLCO [5]. In most cases, an increase in alveolar oxygen pressure provides enough driving pressure to overcome this relative diffusion defect $[2,8]$. However, severe forms of pulmonary arteriovenous dilatation are poorly responsive to administration of $100 \%$ oxygen [9] .

Elevated oestrogen production is a hallmark of pregnancy, and can cause dilation, proliferation and congestion of blood vessels [10]. Varicosities can occur in pregnant females, and increase in intensity between 10 and 32 weeks of gestation, through increased circulating blood volume and cardiac output. However, most of these vascular changes regress postpartum [11].

A similar diagnosis has been reported previously in one case [12]. In this previous case, hypoxaemia was mild and rapidly improved after delivery. By contrast, in our patient, the worsening hypoxaemia, the results of the hyperoxia tests and contrast echocardiography during the late course of pregnancy and early phase after delivery were consistent with marked worsening of the intrapulmonary shunts and a much slower, but almost complete, recovery.

@ERSpublications

Hypoxaemia during pregnancy may be due to a true shunt related to transient pulmonary arteriovenous dilatation http://ow.ly/AjOUr

Matthieu Veil-Picard ${ }^{1}$, Julie Cattin ${ }^{2}$, Romain Chopard ${ }^{3}$, François Schiele ${ }^{3}$, Didier Riethmuller ${ }^{2}$, Jean-Charles Dalphin ${ }^{1,4}$ and Bruno Degano ${ }^{5}$

${ }^{1}$ Dept of Respiratory Diseases, University Hospital, Besançon, France. ${ }^{2}$ Dept of Obstetrics, University Hospital, Besançon, France. ${ }^{3}$ Dept of Cardiology and EA3920, University Hospital, Besançon, France. ${ }^{4}$ UMR CNRS ChronoEnvironnement, 
Université de Franche-Comté, Besançon, France. ${ }^{5}$ Dept of Physiology and EA 3920, University Hospital, Besançon, France.

Correspondence: Bruno Degano, Explorations Fonctionnelles - Physiologie, CHU de Besançon, Boulevard Fleming, 25000 Besançon, France. E-mail: bruno.degano@univ-fcomte.fr

Received: May 132014 | Accepted after revision: June 152014

Conflict of interest: None declared.

Provenance: Submitted article, peer reviewed.

\section{References}

Hegewald MJ, Crapo RO. Respiratory physiology in pregnancy. Clin Chest Med 2011; 32: 1-13.

Melot C, Naeije R. Pulmonary vascular diseases. Compr Physiol 2011; 1: 593-619.

Cutts BA, Dasgupta D, Hunt BJ. New directions in the diagnosis and treatment of pulmonary embolism in pregnancy. Am J Obstet Gynecol 2013; 208: 102-108.

4 Pernot J, Puzenat E, Magy-Bertrand N, et al. Detection of interstitial lung disease in systemic sclerosis through partitioning of lung transfer for carbon monoxide. Respiration 2012; 84: 461-468.

5 Degano B, Mittaine M, Guenard H, et al. Nitric oxide and carbon monoxide lung transfer in patients with advanced liver cirrhosis. J Appl Physiol 2009; 107: 139-143.

6 Swinburne AJ, Fedullo AJ, Gangemi R, et al. Hereditary telangiectasia and multiple pulmonary arteriovenous fistulas. Clinical deterioration during pregnancy. Chest 1986; 89: 459-460.

7 Degano B, Mittaine M, Herve P, et al. Nitric oxide production by the alveolar compartment of the lungs in cirrhotic patients. Eur Respir J 2009; 34: 138-144.

8 Wagner PD, Rodriguez-Roisin R. Clinical advances in pulmonary gas exchange. Am Rev Respir Dis 1991; 143: 883-888.

9 Rodriguez-Roisin R, Krowka MJ. Hepatopulmonary syndrome - a liver-induced lung vascular disorder. $N$ Engl $J$ Med 2008; 358: 2378-2387.

10 Degano B, Mourlanette P, Valmary S, et al. Differential effects of low and high-dose estradiol on airway reactivity in ovariectomized rats. Respir Physiol Neurobiol 2003; 138: 265-274.

11 Tunzi M, Gray GR. Common skin conditions during pregnancy. Am Fam Physician 2007; 75: 211-218.

12 Wilmshurst P, Jackson P. Arterial hypoxemia during pregnancy caused by pulmonary arteriovenous microfistulas. Chest 1996; 110: 1368-1369.

\section{Pulmonary hypertension associated with Whipple disease}

To the Editor:

Whipple disease is a rare multi-systemic disorder caused by Thropheryma whipplei, a Gram-positive bacillus. Gastrointestinal manifestations are the most frequent, but many other organs may be involved $[1,2]$. Pulmonary hypertension $(\mathrm{PH})$ associated with Whipple disease is extremely rare, and only a few isolated cases have been reported. We present two patients with $\mathrm{PH}$-Whipple disease with a positive vasodilator test and excellent response to antibiotic therapy.

\section{Patient 1}

A 72-year-old Caucasian male with an 8-month history of intermittent abdominal pain and diarrhoea presented with dyspnoea on exertion that had progressed in the last 4 months (functional class III) and two-pillow orthopnoea to keep his head at $30^{\circ}$. He reported weight loss of $6 \mathrm{~kg}$ during the preceding 8 months. A colonoscopy performed 15 days before showed only nonspecific inflammation. Past medical history included mild chronic obstructive pulmonary disease treated with ipratropium. Physical examination revealed hypotension (systolic arterial pressure $90 \mathrm{mmHg}$ ) and bibasilar rales but not lower leg oedema. Laboratory data showed haemoglobin of $94 \mathrm{~g} \cdot \mathrm{L}^{-1}$ and albumin of $19 \mathrm{~g} \cdot \mathrm{L}^{-1}$. Antinuclear antibodies, rheumatoid factor and a HIV test were negative. A chest computed tomography (CT) with embolism protocol demonstrated bilateral pleural and pericardial effusions and mediastinal adenopathy. No emboli were found in the pulmonary arteries. Abdominal CT showed mild ascites and multiple small lymph nodes. Gastroduodenoscopy demonstrated diffuse inflammation in the duodenum. Small bowel biopsies showed typical periodic acid-Schiff (PAS)-positive diastase-resistant histiocytes. Several days later PCR confirmed the presence of T. whipplei. Transthoracic echocardiography showed normal left cavities with a 